\title{
The three-term recursion for Chebyshev polynomials is mixed forward-backward stable
}

\author{
Alicja Smoktunowicz • Agata Smoktunowicz • \\ Ewa Pawelec
}

Received: 1 February 2014 / Accepted: 25 September 2014 / Published online: 12 November 2014 (C) The Author(s) 2014. This article is published with open access at Springerlink.com

\begin{abstract}
This paper provides an error analysis of the three-term recurrence relation (TTRR) $T_{n+1}(x)=2 x T_{n}(x)-T_{n-1}(x)$ for the evaluation of the Chebyshev polynomial of the first kind $T_{N}(x)$ in the interval $[-1,1]$. We prove that the computed value of $T_{N}(x)$ from this recurrence is very close to the exact value of the Chebyshev polynomial $T_{N}$ of a slightly perturbed value of $x$. The lower and upper bounds for the function $C_{N}(x)=\left|T_{N}(x)\right|+\left|x T_{N}^{\prime}(x)\right|$ are also derived. Numerical examples that illustrate our theoretical results are given.
\end{abstract}

Keywords Chebyshev polynomials $\cdot$ Error analysis $\cdot$ Roots of polynomials

Mathematics Subject Classifications (2010) 65G50 · 65D20 · 65L70

\section{Introduction}

This paper proves the numerical stability of the three-term recurrence relation (TTRR) of Chebyshev polynomials of the first kind $\left(T_{n}(x)\right)$ :

$$
T_{n}(x)=2 x T_{n-1}(x)-T_{n-2}(x), n=2,3, \ldots,
$$

The research of Agata Smoktunowicz was funded by ERC grant 320974.

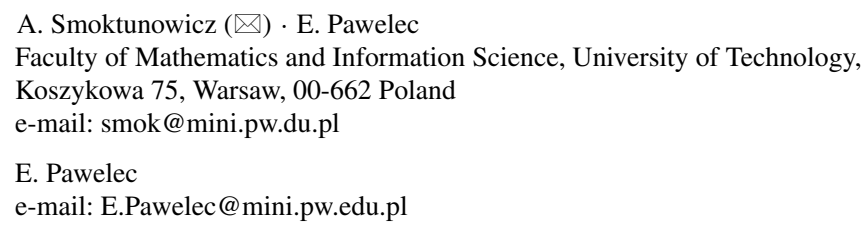


where $T_{0}(x)=1, T_{1}(x)=x$.

This formula often appears in practical applications. The TTRR is used in Forsythe's method for evaluating polynomials in Chebyshev form $p_{N}(x)=$ $\sum_{k=0}^{N} a_{k} T_{k}(x)$. There are several algorithms for evaluating Chebyshev series (see $[1-5,13])$. Clenshaw's and Forsythe's algorithms are recommended. Chebyshev polynomials of the first kind $\left(T_{n}(x)\right)$ are widely used in many applications (see [6, $8,10,14])$. A desirable property for algorithms is numerical stability (see $[12,17]$ ). The term stability is sometimes used to refer to the forward or backward analysis of the algorithm. An error analysis of Clenshaw's algorithm in the general case was first provided by D. Elliott in [9]. See also [2-5, 7, 11, 15], where the authors present the forward error bounds for the evaluation of $p_{N}(x)=\sum_{k=0}^{N} a_{k} T_{k}(x)$.

In this paper we study the mixed forward-backward stability of the TTRR (see [12], Section 1.5). A precise definition of mixed forward-backward stability is now given.

Definition 1 An algorithm $W$ of computing $T_{N}(x)$ is called mixed forwardbackward stable with respect to the data $x$, if the value $\tilde{T}_{N}(x)$ computed by $W$ in floating point arithmetic satisfies

$$
\tilde{T}_{N}(x)=\left(1+\delta_{N}\right) T_{N}\left(\left(1+\Delta_{N}\right) x\right)+\mathcal{O}\left(\epsilon_{M}^{2}\right),\left|\delta_{N}\right|,\left|\Delta_{N}\right| \leq \epsilon_{M} L,
$$

where $L=L(N)$ is a modestly growing function on $N$ and $\epsilon_{M}$ is machine precision.

Simply, the computed value of $T_{N}(x)$ by mixed forward-backward stable algorithm is very close to the exact value of the Chebyshev polynomial $T_{N}$ of a slightly perturbed value of $x$. Throughout this paper, we will ignore the terms of order $\mathcal{O}\left(\epsilon_{M}^{2}\right)$. Notice that (see, e.g., Lemma 4.1 in [18]) that the property (2) is equivalent to

$$
\left|\tilde{T}_{N}(x)-T_{N}(x)\right| \leq \epsilon_{M} L C_{N}(x)+\mathcal{O}\left(\epsilon_{M}^{2}\right),
$$

where

$$
C_{N}(x)=\left|T_{N}(x)\right|+\left|x T_{N}^{\prime}(x)\right|=\left|T_{N}(x)\right|+N\left|x U_{N-1}(x)\right|,
$$

where $U_{N-1}(x)$ denotes the Chebyshev polynomial of the second kind. These polynomials satisfy the recurrence relations

$$
U_{n}(x)=2 x U_{n-1}(x)-U_{n-2}(x), n=2,3, \ldots,
$$

where $U_{0}(x)=1, U_{1}(x)=2 x$.

The paper has been organized as follows. In Section 2, we recall some basic properties of the Chebyshev polynomials. In Section 3, we will use these properties in a derivation of the lower and upper bounds for $C_{n}(x)$. In Section 4, we present the error analysis for the TTR. We prove that the TTRR is mixed forward-backward stable in the sense of (3) (which is equivalent to (2). Finally, in Section 5 we present some numerical experiments performed in MATLAB. 


\section{Preliminaries}

We will need some properties of the Chebyshev polynomials (see [14, 16]). For $-1 \leq x \leq 1$, we have $T_{n}(x)=\cos (n \Theta)$, where $\Theta=\arccos x$ and $U_{n-1}(x)=$ $\sin (n \Theta) / \sin \Theta$ for $0<x<1$.

The following identities hold:

$$
\begin{gathered}
U_{n-1}(x)=\frac{T_{n}{ }^{\prime}(x)}{n}, \\
T_{n}(-x)=(-1)^{n} T_{n}(x), \quad U_{n}(-x)=(-1)^{n} U_{n}(x) .
\end{gathered}
$$

The Chebyshev polynomials of the first kind satisfy the following differential equations;

$$
\left(1-x^{2}\right) T_{n}^{\prime \prime}(x)-x T_{n}^{\prime}(x)+n^{2} T_{n}(x)=0
$$

and

$$
T_{n}^{2}(x)+\frac{1-x^{2}}{n^{2}} T_{n}^{\prime 2}(x)=1 .
$$

The last equality is a consequence of the trigonometric identity $\cos ^{2} n \theta+\sin ^{2} n \theta=1$.

For $-1 \leq x \leq 1$ and $n=0,1, \ldots$ we have the upper bounds

$$
\left|T_{n}(x)\right| \leq\left|T_{n}(1)\right|=1, \quad\left|U_{n}(x)\right| \leq\left|U_{n}(1)\right|=n+1
$$

and for $-1<x<1$

$$
\left|U_{n}(x)\right| \leq \frac{1}{\sqrt{1-x^{2}}} .
$$

The roots $\left(t_{i}\right)$ of $T_{n}(x)$ are distinct and belong to $(-1,1)$ :

$$
t_{i}=\cos \frac{(2 i-1) \pi}{2 n}, \quad i=1,2, \ldots, n .
$$

The roots $\left(u_{i}\right)$ of $T_{n}^{\prime}(x)$ (i.e. the roots of $\left.U_{n-1}(x)\right)$ are:

$$
u_{i}=\cos \frac{i \pi}{n}, \quad i=1,2, \ldots, n-1 .
$$

Then $-1<t_{n}<u_{n-1}<\ldots<u_{1}<t_{1}<1$ and

$$
T_{n}\left(u_{i}\right)=(-1)^{i} \quad i=1,2, \ldots, n-1 .
$$

For $-1 \leq x \leq 1$ and $m=0,1, \ldots$ we get

$$
\left|T_{2 m+1}(x)\right| \leq(2 m+1)|x|, \quad\left|U_{2 m+1}(x)\right| \leq 2(m+1)|x| .
$$

\section{Lower and upper bounds for $C_{n}(x)$}

Since $C_{n}(-x)=C_{n}(x)$ for all $x$, we restrict our considerations to the interval [0, 1]. From (8) it follows that $C_{n}(x) \leq C_{n}(1)=n^{2}+1$ for $0 \leq x \leq 1$. By (11)-(12), we have $C_{n}\left(u_{i}\right)=1$ for $i=1, \ldots, n-1$. If $n$ is odd then $C_{n}(0)=0$ (Figs. 1 and 2). 


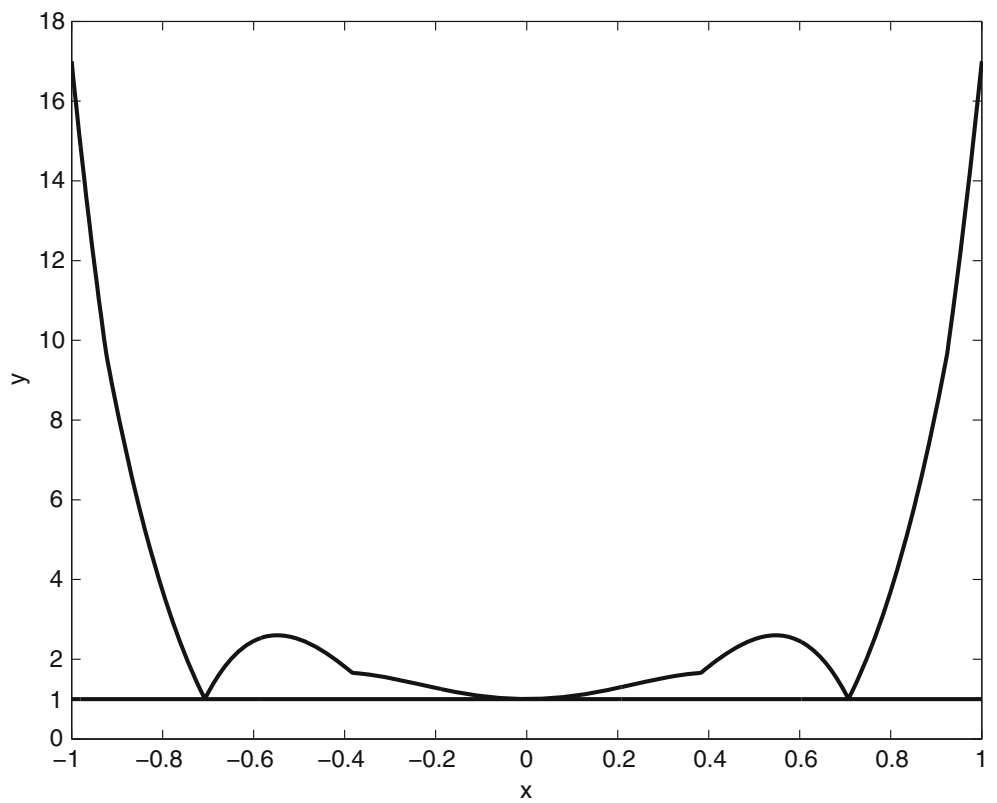

Fig. 1 Plot of $y=C_{4}(x)$

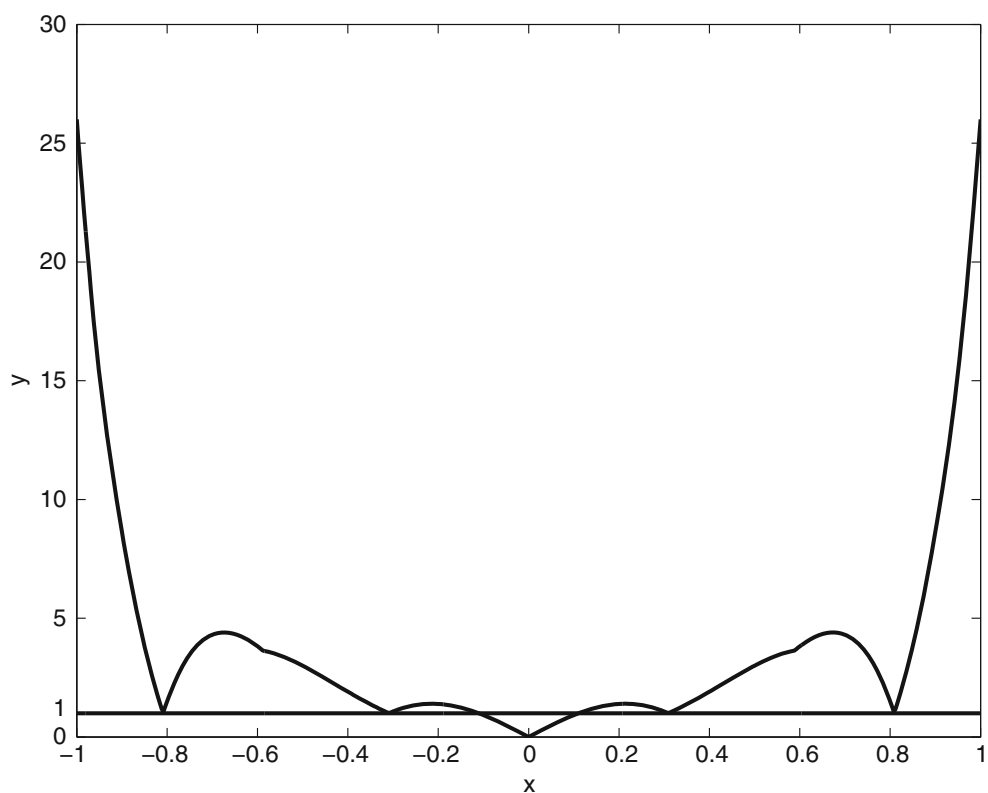

Fig. 2 Plot of $y=C_{5}(x)$ 
Theorem 1 Let $n$ be a natural number. Assume that $s_{n} \leq x \leq 1$, where

$$
s_{n}=\frac{1}{\sqrt{n^{2}+1}} \text {. }
$$

Then we have

$$
C_{n}(x)=\left|T_{n}(x)\right|+\left|x T_{n}^{\prime}(x)\right| \geq 1 .
$$

Proof Notice that the inequality $x^{2} \geq s_{n}^{2}$ is equivalent to $x^{2}\left(n^{2}+1\right) \geq 1$, hence $x^{2} \geq \frac{1-x^{2}}{n^{2}}$. From this and (7) we get

$$
C_{n}^{2}(x) \geq T_{n}^{2}(x)+x^{2} T_{n}^{\prime 2}(x) \geq T_{n}^{2}(x)+\frac{1-x^{2}}{n^{2}} T_{n}^{\prime 2}(x)=1 .
$$

The proof is now complete.

Theorem 2 Let $n$ be a natural number. Assume that $0 \leq x \leq s_{n}$, where $s_{n}$ is defined by (14). Then

(i) $C_{n}(x) \geq n|x|$ for all $n$,

(ii) $C_{n}(x) \geq 1$ for even $n$.

Proof We consider case (i). Clearly, by (14) $1 \geq n^{2} x^{2}$, and since $0 \leq x \leq s_{n}$, we get $C_{n}^{2}(x) \geq T_{n}^{2}(x)+x^{2} T_{n}^{\prime 2}(x) \geq n^{2} x^{2} T_{n}^{2}(x)+x^{2} T_{n}^{\prime 2}(x) \geq x^{2} n^{2}\left(T_{n}^{2}(x)+\frac{1}{n^{2}} T_{n}^{\prime 2}(x)\right)$.

Therefore,

$$
C_{n}^{2}(x) \geq x^{2} n^{2}\left(T_{n}^{2}(x)+\frac{1-x^{2}}{n^{2}} T_{n}^{\prime 2}(x)\right)=x^{2} n^{2},
$$

due to (7). Therefore, $C_{n}(x) \geq n|x|$. This completes the proof of case (i).

Now we consider case (ii). Let $n=2 \mathrm{~m}$. We first prove that $T_{2 m}$ has no roots in $\left(0, s_{2 m}\right)$. By $(10)$, we need to show that

$$
t_{m}=\cos \frac{(2 m-1) \pi}{4 m}>s_{2 m} .
$$

Notice that

$$
t_{m}=\cos \left(\frac{\pi}{2}-\frac{\pi}{4 m}\right)=\sin \frac{\pi}{4 m} .
$$

Since $0<\Theta<\tan \Theta$ for all $0<\Theta<\frac{\pi}{2}$, we have $\tan ^{2} \Theta>\Theta^{2}$. From this it follows that $\sin ^{2} \Theta>\frac{\Theta^{2}}{1+\Theta^{2}}$. Substituting $\Theta=\pi / 4 m$ in the above inequality leads to

$$
t_{m}^{2}>\frac{\pi^{2}}{16 m^{2}+\pi^{2}}>\frac{1}{4 m^{2}+1}=s_{2 m}^{2},
$$

so $t_{m}>s_{2 m}$. This finishes the proof of (16).

We see that $T_{2 m}$ has no roots in $\left(0, s_{2 m}\right)$. Moreover, $T_{2 m}(0)=(-1)^{m}$ and $T_{2 m}^{\prime}(0)=0$. We conclude from (10)-(11) that 0 is the only root of $T_{2 m}^{\prime}$ in the interval $\left(-s_{2 m}, s_{2 m}\right)$. Notice that $T_{2 m}$ and $T_{2 m}^{\prime \prime}$ are even, i.e. $T_{2 m}(-x)=T_{2 m}(x)$ and 
$T_{2 m}^{\prime \prime}(-x)=T_{2 m}^{\prime \prime}(x)$ for all $x . T_{2 m}^{\prime}$ is odd, that is, $T_{2 m}^{\prime}(-x)=-T_{2 m}^{\prime}(x)$. Thus we see that the polynomials $T_{2 m}$ and $T_{2 m}^{\prime}$ do not change the signs in $\left(0, s_{2 m}\right)$.

More precisely, if $m$ is even, then for all $0<x<s_{2 m}$ we have $T_{2 m}(x)>0$ and $T_{2 m}^{\prime}(x)<0$, hence $C_{2 m}(x)=T_{2 m}(x)-x T_{2 m}^{\prime}(x)$. Similarly, if $m$ is odd then $T_{2 m}(x)<0$ and $T_{2 m}^{\prime}(x)>0$, so $C_{2 m}(x)=-T_{2 m}(x)+x T_{2 m}^{\prime}(x)$. We see that $C_{2 m}^{\prime}(x)=-T_{2 m}^{\prime \prime}(x)$ if $m$ is even and $C_{2 m}^{\prime}(x)=T_{2 m}^{\prime \prime}(x)$ otherwise.

By (6) for $n=2 m$, we obtain the formula

$$
\left(1-x^{2}\right) T_{2 m}^{\prime \prime}(x)=x T_{2 m}^{\prime}(x)-2 m^{2} T_{2 m}(x) .
$$

We see that for all $0<x<s_{2 m}$ we have $T_{2 m}^{\prime \prime}(x)<0$ if $m$ is even and $T_{2 m}^{\prime \prime}(x)>0$ if $m$ is odd. We conclude that $C_{2 m}^{\prime}(x)>0$ for any $m$, so $C_{2 m}(x)$ is increasing in the interval $\left(0, s_{2 m}\right)$. This gives the lower bound $C_{2 m}(x) \geq C_{2 m}(0)=1$. The proof of our theorem is now complete.

\section{Error analysis}

We analyze the rounding errors in the TTRR. We start with the following lemma.

Lemma 1 Let $\tilde{T}_{n}(x)$ denote the quantities computed by the TTRR in floating point arithmetic $f l$ with machine precision $\epsilon_{M}$. Assume that $x$ is exactly representable in $f l$ $(f l(x)=x)$ and $x \in[-1,1]$. Then

$$
\left|\tilde{T}_{N}(x)-T_{N}(x)\right| \leq \epsilon_{M} E_{N}(x)+\mathcal{O}\left(\epsilon_{M}^{2}\right),
$$

where

$$
E_{N}(x)=\sum_{n=2}^{N}\left(2|x|\left|T_{n-1}(x)\right|+\left|T_{n}(x)\right|\right)\left|U_{N-n}(x)\right| .
$$

Proof Note that $\tilde{T}_{0}(x)=1, \tilde{T}_{1}(x)=x$ and for $n=2,3, \ldots$ we have

$$
\tilde{T}_{n}(x)=\left(2 x \tilde{T}_{n-1}(x)\left(1+\alpha_{n}\right)-\tilde{T}_{n-2}(x)\right)\left(1+\beta_{n}\right), \quad\left|\alpha_{n}\right|,\left|\beta_{n}\right| \leq \epsilon_{M} .
$$

Therefore,

$$
\tilde{T}_{n}(x)=2 x \tilde{T}_{n-1}(x)-\tilde{T}_{n-2}(x)+\xi_{n}, \quad \xi_{n}=2 x \tilde{T}_{n-1}(x) \alpha_{n}+\frac{\beta_{n}}{1+\beta_{n}} \tilde{T}_{n}(x) .
$$

Let $e_{n}=\tilde{T}_{n}(x)-T_{n}(x)$. We observe that $e_{0}=e_{1}=0$ and $e_{n}=2 x e_{n-1}-$ $e_{n-2}+\xi_{n}$ for $n=2,3, \ldots, N$. From this it follows that $e_{N}=\tilde{T}_{N}(x)-T_{N}(x)=$ $\sum_{n=2}^{N} U_{N-n}(x) \xi_{n}$. Therefore, $\left|e_{N}\right| \leq \sum_{n=2}^{N}\left|U_{N-n}(x)\right|\left|\xi_{n}\right|$. This, together with (19), leads to

$$
\left|\xi_{n}\right| \leq \epsilon_{M}\left(2|x|\left|T_{n-1}(x)\right|+\left|T_{n}(x)\right|\right)+\mathcal{O}\left(\epsilon_{M}^{2}\right)
$$

hence

$$
\left|e_{N}\right| \leq \epsilon_{M} \sum_{n=2}^{N}\left(2|x|\left|T_{n-1}(x)\right|+\left|T_{n}(x)\right|\right)\left|U_{N-n}(x)\right|+\mathcal{O}\left(\epsilon_{M}^{2}\right) .
$$


The proof of Lemma 1 is now complete.

Now our task is to bound $E_{N}(x)$.

Theorem 3 Let $E_{N}(x)$ be defined by (18). Then we have

$$
E_{N}(x) \leq E_{N}(1)=\frac{3 N(N-1)}{2} \quad \text { for } x \in[-1,1]
$$

and

$$
E_{N}(x) \leq \frac{3(N-1)}{\sqrt{1-x^{2}}} \quad \text { for } x \in(-1,1) .
$$

If $N$ is odd and $|x| \leq s_{N}=\frac{1}{\sqrt{N^{2}+1}}$ then

$$
E_{N}(x) \leq \frac{5(N-1)(N+7)}{8}|x| .
$$

Proof It is obvious that for every $x \in[-1,1]$ we have

$$
E_{N}(x) \leq E_{N}(1)=3 \sum_{n=2}^{N}(N-n+1)=\frac{3 N(N-1)}{2} .
$$

This completes the proof of (22).

If $-1<x<1$ then by (8)-(9) and (18) we get

$$
E_{N}(x) \leq 3 \sum_{n=2}^{N} \frac{1}{\sqrt{1-x^{2}}}=\frac{3(N-1)}{\sqrt{1-x^{2}}} .
$$

This finishes the proof of (23).

Now assume that $N$ is odd and $|x| \leq s_{N}$. We rewrite (18) as follows

$$
\left.E_{N}(x)=A_{N}(x)+B_{N}(x)\right),
$$

where

$$
\begin{gathered}
A_{N}(x)=2|x| \sum_{n=2}^{N}\left|T_{n-1}(x)\right|\left|U_{N-n}(x)\right|, \\
B_{N}(x)=\sum_{n=2}^{N}\left|T_{n}(x)\right|\left|U_{N-n}(x)\right| .
\end{gathered}
$$

Notice that (9) gives

$$
\left|U_{k}(x)\right| \leq \frac{1}{\sqrt{1-s_{N}^{2}}} \leq \frac{3}{2} \text { for }|x| \leq s_{N}, \quad k=0,1, \ldots
$$

This, together with the inequality $\left|T_{n-1}(x)\right| \leq 1$, gives

$$
A_{N}(x) \leq 3|x|(N-1) \text {. }
$$


Table 1 The error (33) for the TTRR

\begin{tabular}{lllllllll}
\hline$N$ & 8 & 16 & 32 & 64 & 128 & 256 & 512 & 1024 \\
\hline Error (33) & 5.25 & 11.00 & 21.78 & 35.00 & 66.00 & 165.00 & 280.75 & 679.62 \\
\hline
\end{tabular}

To estimate $B_{N}(x)$ for $N=2 m+1$, we split it as follows:

$$
B_{N}(x)=\sum_{k=1}^{m}\left|T_{2 k}(x)\right|\left|U_{N-2 k}(x)\right|+\sum_{k=1}^{m}\left|T_{2 k+1}(x)\right|\left|U_{N-(2 k+1)}(x)\right| .
$$

Note that (13) implies the following upper bounds (for the polynomials of the odd degrees):

$$
\left|U_{N-2 k}(x)\right| \leq(N-2 k+1)|x|, \quad\left|T_{2 k+1}(x)\right| \leq(2 k+1)|x| .
$$

From this and (28) it follows that

$$
B_{N}(x) \leq \sum_{k=1}^{m}(N-2 k+1)|x|+\frac{3}{2} \sum_{k=1}^{m}(2 k+1)|x| .
$$

The last inequality, together with (25) and (29), leads to

$$
E_{N}(x) \leq\left(3(N-1)+m(N-m)+\frac{3}{2} m(m+2)\right)|x| .
$$

Since $m=(N-1) / 2$, we immediately get (24).

The bounds $(22,23)$ are not new, see Barrio $[2,5]$. To our knowledge, the bound (24) is however new, and allows us to prove the mixed forward-backward stability of the TTRR for all $x \in[-1,1]$.

By Theorems 1-3 and Lemma 1 we obtain the following theorem.

Theorem 4 The TTRR is mixed forward-backward stable in $[-1,1]$. For every $N \geq$ 2 we have

$$
\left|\tilde{T}_{N}(x)-T_{N}(x)\right| \leq \epsilon_{M} \frac{3 N(N-1)}{2} C_{N}(x)+\mathcal{O}\left(\epsilon_{M}^{2}\right) \quad \text { for } x \in[-1,1]
$$

and

$$
\left|\tilde{T}_{N}(x)-T_{N}(x)\right| \leq \epsilon_{M} 7(N-1) C_{N}(x)+\mathcal{O}\left(\epsilon_{M}^{2}\right) \quad \text { for } x \in[-0.9,0.9] .
$$

Moreover, if $N$ is odd and $|x| \leq s_{N}=\frac{1}{\sqrt{N^{2}+1}}$, then

$$
\left|\tilde{T}_{N}(x)-T_{N}(x)\right| \leq \epsilon_{M} \frac{5(N-1)(N+7)}{8 N} C_{N}(x)+\mathcal{O}\left(\epsilon_{M}^{2}\right) .
$$

We see that $L(N)$ in $(2)$ is of order $N$ in $(-1,1)$ for $x$ not to close to \pm 1 and of order $N^{2}$ near \pm 1 . 
Table 2 The relative error $R_{N}(x)$

\begin{tabular}{lllllll}
\hline$N$ & 10 & $10^{2}$ & $10^{3}$ & $10^{4}$ & $10^{5}$ & $10^{6}$ \\
\hline$R_{N}(0)$ & 5 & 50 & 500 & $5 \cdot 10^{3}$ & $5 \cdot 10^{4}$ & $5 \cdot 10^{5}$ \\
$R_{N}(0.5)$ & 1.63 & 1.96 & 1.99 & 1.99 & 2.00 & 2.00 \\
$R_{N}(0.9)$ & 1.33 & 1.61 & 1.48 & 1.50 & 1.48 & 1.75 \\
$R_{N}(\cos (2 \pi / N))$ & 14.70 & $1.51 \cdot 10^{3}$ & $1.51 \cdot 10^{5}$ & $1.50 \cdot 10^{7}$ & $1.62 \cdot 10^{7}$ & $4.33 \cdot 10^{6}$ \\
$R_{N}(\cos (\pi / N))$ & 29.41 & $3.03 \cdot 10^{3}$ & $3.03 \cdot 10^{5}$ & $2.91 \cdot 10^{7}$ & $8.24 \cdot 10^{6}$ & $1.06 \cdot 10^{5}$ \\
$R_{N}(1)$ & 1.33 & 1.48 & 1.49 & 1.49 & 1.50 & 1.50 \\
\hline
\end{tabular}

\section{Numerical tests}

To illustrate our results, we present numerical tests in MATLAB with machine precision $\epsilon_{M}=2^{-52} \approx 2.2 \cdot 10^{-16}$. We compare the results computed by the TTRR with the exact values of the Chebyshev polynomial $T_{N}(x)$. They were obtained by implementing the TTRR in high precision using the VPA (Variable Precision Arithmetic) function from MATLAB's Symbolic Math Toolbox, and then rounded to 16th decimal digits. We compute the relative error

$$
e_{N}=\frac{\max _{x \in S}\left|T_{N}(x)-\tilde{T}_{N}(x)\right|}{\epsilon_{M}} .
$$

Here $S$ consists of the equally spaced checkpoints $t_{1}, t_{2}, \ldots, t_{201}$ from the interval $[-1,1]$, i.e. $t_{i}=-1+(i-1) / 100$ for $i=1,2, \ldots, 201$ (Table 1). In order to show that our theoretical bounds are realistic, we evaluated $R_{N}(x)=E_{N}(x) / C_{N}(x)$ for particular values of $x$. It is clear that near $x= \pm 1$ the values of $R_{N}$ are of order $N^{2}$ for large $N$ (Table 2). For example, if $\hat{x}=u_{1}=\cos (\pi / N)$ (the root of $T_{N}^{\prime}(x)$ ) then $\hat{x}$ is very close to 1 , so $E_{N}(\hat{x}) \approx E_{N}(1)=\frac{3 N(N-1)}{2}$. However, $C_{N}(\hat{x})=1$, so $R_{N}(\hat{x}) \approx \frac{3 N(N-1)}{2}$. Similar conclusion can be found in [2] for a class of parallel algorithms to evaluate Chebyshev series.

Acknowledgments The authors are grateful to the referees for carefully reading our manuscript and for their fruitful suggestions and remarks, which helped to improve the paper.

Open Access This article is distributed under the terms of the Creative Commons Attribution License which permits any use, distribution, and reproduction in any medium, provided the original author(s) and the source are credited.

\section{References}

1. Bakhvalov, N.S.: The stable calculation of polynomial values. J. Comp. Math. Math. Phys. 11, 15681574 (1971)

2. Barrio, R.: Stability of parallel algorithms to evaluate Chebyshev series. Comput. Math. Appl. 41, 1365-1377 (2001)

3. Barrio, R.: Rounding error bounds for the Clenshaw and Forsythe algorithms for the evaluation of orthogonal series. J. Comput. Appl. Math. 138, 185-204 (2002) 
4. Barrio, R.: A unified rounding error bound for polynomial evaluation. Adv. Comput. Math. 19(4), 385-399 (2003)

5. Barrio, R., Jiang, H., Serrano, S.: A general condition number for polynomials. SIAM J. Numer. Anal. 51(2), 1280-1294 (2013)

6. Berrut, J.-P., Trefethen, L.N.: Barycentric Lagrange interpolation. SIAM Rev. 46(3), 501-517 (2004)

7. Deuflhard, P.: On algorithm for the summation of certain special functions. Computing 17, 37-48 (1976)

8. Driscoll, T.A., Hale, N., Trefethen, L.N. (eds.): Chebfun Guide. Pafnuty Publications, Oxford (2014)

9. Elliott, D.: Error analysis of an algorithm for summing certain finite series. J. Austral. Math. Soc. 8, 213-221 (1968)

10. Gautschi, W.: Orthogonal Polynomials: Computation and Approximation (Numerical Mathematics and Scientific Computation). Oxford University Press (2004)

11. Gentleman, W.M.: An error analysis of Goertzel's (Watt's) method for computing Fourier coefficients. Comput. J. 12, 160-165 (1969)

12. Higham, N.J.: Accuracy and Stability of Numerical Algorithms. SIAM, Philadelphia (1996)

13. Koepf, W.: Efficient computation of Chebyshev polynomials. In: Wester, M.J. (ed.) Computer Algebra Systems: A Practical Guide, pp. 79-99. Wiley, New York (1999)

14. Paszkowski, S.: Numerical Applications of Chebyshev Polynomials. Warsaw, (in Polish) (1975)

15. Smoktunowicz, A.: Backward stability of Clenshaw's algorithm. BIT 42(3), 600-610 (2002)

16. Szegö, G.: Orthogonal Polynomials, rev. ed. New York (1959)

17. Wilkinson, J.H.: The Algebraic Eigenvalue Problems. Oxford University Press (1965)

18. Woźniakowski, H.: Numerical stability for solving nonlinear equations. Numer. Math. 27, 373-390 (1977) 600-610 